\title{
Le personnage du collectionneur au XIXe siècle : de l'excentrique à l'amateur distingué
}

\section{Dominique Pety}

\begin{abstract}
The collector figure, analyzed from 1840 to the end of the 19th century in a large variety of literary and non-literary texts, evolves from an excentric and monomaniac type leading a cloistered life, to a fashionable connoisseur, very ken on exhibiting his possessions. This change in representation proves collecting being totally reconsidered in the period, as well as values associated to it: aristocratic and traditional values of art and art of life versus new republican values of knowledge and science. Private collections, formerly aimed at personal delight, now respond to the duty of national heritage preservation.
\end{abstract}

\section{Résumé}

Le personnage du collectionneur, analysé de 1840 à la fin du siècle dans un large choix de textes littéraires et non littéraires, passe de l'excentrique monomane reclus dans sa demeure à l'amateur mondain, qui exhibe ses possessions. Cette évolution de la représentation témoigne d'une spectaculaire réévaluation de la pratique, mais aussi de la mutation des valeurs associées à la collection: valeurs aristocratiques traditionnelles de l'art et de l'art de vivre, valeurs républicaines nouvelles du savoir et de la science. Les collections privées, jadis sources de délectation personnelle, participent désormais au devoir de préservation du patrimoine national.

\section{Citer ce document / Cite this document :}

Pety Dominique. Le personnage du collectionneur au XIXe siècle : de l'excentrique à l'amateur distingué. In: Romantisme, 2001, n¹12. La collection. pp. 71-81;

doi : https://doi.org/10.3406/roman.2001.6173

https://www.persee.fr/doc/roman_0048-8593_2001_num_31_112_6173

Fichier pdf généré le 02/04/2018 


\section{Le personnage du collectionneur au XIX ${ }^{\mathrm{e}}$ siècle : de l'excentrique à l'amateur distingué}

Des années 1840 à la fin du XIX siècle, le personnage du collectionneur, tel qu'il est mis en scène dans les chroniques journalistiques, dans divers genres littéraires, tel qu'il se dessine aussi en creux dans les préambules de discours plus spécialisés, change du tout au tout, passant du marginal, au physique et au costume disgraciés, au mondain ou à l'aristocrate. Cette transformation de la représentation témoigne des mutations intervenues dans la réalité culturelle et sociale, avec néanmoins quelques décalages: dans les ventes publiques, les prix flambent dès les années 1850-1860, comme l'indiquent des témoignages ponctuels comme le Journal des Goncourt ou l'Annuaire des artistes et des amateurs (1860-62) de Paul Lacroix. La collection commence sous le Second Empire à devenir un fait de mode, même si elle n'est enregistrée comme telle qu'après 1870 , au moment où elle se déchaîne ${ }^{1}$. D'autres distorsions sont aussi à relever: le portrait littéraire du collectionneur en excentrique vers 1840 cache des réalités plus larges; de grands collectionneurs, publiquement reconnus, existent à cette date, comme Du Sommerard dont l'État achète la collection en 1843, comme Sauvageot, qui lègue la sienne au Louvre en 1856, sans parler des grandes fortunes comme Hertford ou Rothschild. Peut-être eût-il fallu aux personnages fictifs confronter quelques collectionneurs réels.

Ce qui nous importait cependant était moins de brosser le tableau d'une réalité culturelle, que de mettre en scène le débat d'idées suscité au fil du siècle par la pratique de la collection. En essayant de dresser conjointement la typologie des représentations du collectionneur et celle des discours sur la collection, selon le genre littéraire (de la physiologie au roman psychologique, en passant par le récit excentrique ou la nouvelle fantastique), ou plus largement le dispositif d'écriture auquel ils ont recours (de la chronique au dictionnaire), nous avons voulu suivre le renversement axiologique qu'ils opèrent, la mutation radicale des valeurs associées à la pratique de la collection. Cette évolution, au cours de laquelle vont s'affronter les valeurs aristocratiques traditionnelles de l'art et de l'otium, et les valeurs républicaines nouvelles du savoir et de l'effort, s'inscrit donc dans une histoire plus générale des idées et des mentalités. Si la collection devient un fait social dominant au XIX'e siècle, c'est peut-être parce qu'elle a su incarner tour à tour la vocation pédagogique et didactique du discours culturel républicain, et le désir de repli dans l'art et la sphère privée, né de l'angoisse d'un monde nouveau.

1. C'est surtout après 1870 qu'on prend conscience d'une rupture, et qu'on évoque avec nostalgie un âge d'or de la collection: faible demande, marchandise abondante et prix bas. Voir Burty, Paris-Guide (1867), Goncourt La Maison d'un artiste (1881), Monselet, préface à Paul Eudel, L'Hôtel Drouot et la curiosité en 1883 (1884): époque mythique des trouvailles miraculeuses chez les ferrailleurs et les fripiers. 


\section{L'excentrique: l'imaginaire social des années 1840}

C'est d'abord dans les physiologies des années 1840 qu'apparaît le personnage du collectionneur. Mais il y occupe une place encore discrète, qui reflète bien sa part réduite dans l'imaginaire social, à l'opposé de types comme «le bourgeois» ou «l'artiste », maintes fois décrits : absent de recueils comme Paris ou le Livre des cent-et-un (1831-1834), ou Le Diable à Paris (1845), on ne le rencontre que dans Les Français peints par eux-mêmes, quoiqu'à deux reprises: dans une monographie d'Horace de Viel-Castel («Les collectionneurs», 1840), dans une subdivision de la physiologie du «rentier» par Balzac (1841). Dans les deux cas, c'est un individu anormal et asocial qui est décrit. La Bruyère avait déjà souligné la relation exclusive qui unit le collectionneur à sa collection, et le rend indifférent à la société et à sa propre famille. Mais ce qui n'était alors qu'une forme de «l'assujettissement aux modes », faiblesse répandue et révélatrice de l'humaine vanité, devient vers 1840 une pathologie singulière: le collectionneur est non seulement un passionné, mais aussi un «fou», et un «monomane». Son portrait physique le trahit: le «collectionneur pur-sang» est

un petit vieillard sec, ridé, râpé, retapé, enveloppé d'une sorte de grande redingote brunâtre, la tête recouverte d'une clémentine de soie noire, par-dessus laquelle se prélasse un énorme chapeau de couleur douteuse, gras des bords, gras de la forme, gras du galon, gras de la coiffe, gras de partout, et qui depuis trente ans, assiste régulièrement avec son maître à toutes les ventes [...].

Balzac dit aussi le «vêtement peu soigné», les «cheveux épars», la «figure détruite» du rentier collectionneur, et le qualificatif de "toqué » que lui appliquent ses voisins. Mais qualifiant la collection de «passion réelle, définie, reconnue chez les anthropomorphes qui habitent les grandes villes », il la reconnaît comme un fait anthropologique avant la lettre. Elle tourne ici à vide, elle s'applique à des «riens» (maître mot de cette physiologie), elle n'est pas rachetée par son objet, et il peut en examiner le mécanisme mis à nu. Dans Le Cousin Pons (1847), le roman lui permettra d'inscrire les composantes du stéréotype dans une logique psychologique (la collection est un substitut à l'affection manquante, une compensation à l'isolement subi). Le collectionneur se transforme en victime, la collection devient un refuge et une thérapie.

Avant d'analyser plus avant l'évolution du type, il faut envisager sa présence dans d'autres genres, comme le récit excentrique. Le collectionneur, par sa figure d'original, aura sa place dans cet anti-roman (voir notamment la variété particulière du bibliophile dans les textes de Nodier). En outre, le récit excentrique, dans sa mise en cause de la linéarité romanesque, inclut volontiers la liste, transcription élémentaire de la collection, qui reproduit dans l'écriture ses juxtapositions et sa disparate. On en a l'exemple le plus clair dans Les Aventures de $M$. Bric-à-brac, roman zoologique, archéologique et paléontologique (1842) d'É. de La Bédollière et $\mathrm{P}$. Bernard. Le héros, au nom explicite, est cette fois un fou avéré qui décide «de se séquestrer du commerce des hommes, de se claquemurer au milieu de ses chères antiquités ». Une succession de courts récits se déploie, centrés sur les pièces de la collection, et le livre se clôt sur un inventaire d'objets incongrus.

La marginalité du collectionneur lui donne aussi un emploi dans le conte ou la nouvelle fantastiques. Il n'est plus alors présenté comme une victime, mais comme un prédateur. Dans Le Fuenzès (1847), confiant le portrait de son collectionneur à des artistes, Champfleury le livre d'emblée à la charge: œil vairon, nez en vrille, accen- 
tuation des cordes du cou, mains d'avares, la laideur est assurée, mais elle se clôt sur cette note inquiétante:

Voyez-vous le dandinement du corps, une manie particulière aux bêtes enfermées et aux idiots, répliqua le dessinateur. Cet homme-là, je le connais, je me le rappelle maintenant... [...] j'ai rencontré cet original dans un roman d'Hoffmann.

La suite du texte confirmera cette présomption: le collectionneur désire ardemment un tableau qui portera malheur à tous ses acquéreurs et l'acculera lui-même au suicide. Dans la même veine, les Goncourt publient en 1852 une nouvelle mettant en scène une étrange marchande de curiosités, «vieille petite femme vêtue, des pieds à la tête, de noir, et propre comme pourrait l'être une sorcière hollandaise », «diabolique petite créature » qui jette finalement un sort à un rival pour acquérir une pièce manquante ${ }^{2}$. En 1862, Champfleury poursuit ce portrait du collectionneur: le Gardilanne du Violon de faïence a, comme un serpent, des «sillons verdâtres sur une peau jaune et luisante », il déploie dans sa chasse aux objets d'art une «astuce diabolique » avec les marchands. Mais la collection elle-même est une activité maléfique et mortelle: inoculée à Dalègre, elle transforme l'honnête homme et le bon vivant en malade et en fou, et lui fait rompre avec la société: la collection est toujours une pratique déviante, mais elle se répand, puisqu'elle commence à contaminer le bon bourgeois...

Symptomatique de cette évolution est le transfert des genres: le collectionneur va commencer à habiter le roman réaliste. Il conserve certes des traits du stéréotype, mais nuancés et minorés. Le collectionneur de Renée Mauperin (1864) est un personnage d'arrière-plan, ridiculisé par son affolement dans une enchère et son plaisir de satyre à la vue d'une nouvelle estampe, mais il introduit l'héroïne des Goncourt dans un lieu qui est en passe de devenir un espace social à la mode: l'Hôtel Drouot. Renée $y$ vient pour trouver un mari, sur le conseil de sa mère («c'est que, vois-tu, maintenant, ces expositions, c'est si couru»). De façon similaire, le roman de Jules Claretie, Un assassin (1866), met en scène, d'une part, un personnage de collectionneur, désigné traditionnellement comme un original, mais rendu atypique par son bon cœur et sa philanthropie; d'autre part, une collection, dont la description occupe une place assez voyante (le centre du livre), et qui rendrait possible le mariage du héros avec sa cousine, et son retour à une vie apaisée et normalisée. Cela n'aura pas lieu, mais la collection est apparue un instant comme la condition d'une rédemption. Elle entre désormais dans la liste des accessoires du réalisme, elle commence à constituer une caution de vraisemblance du récit dans lequel elle figure, parce qu'elle s'est également répandue dans la vie sociale.

\section{Entre caricature et réalité: à l'hôtel des ventes}

Dans les années 1860, le discours ironique des physiologies et leur stéréotype du collectionneur maniaque persiste, mais dans un genre hybride, celui des chroniques de l'hôtel des ventes, qui donne sur le ton de la satire une information qui n'est plus destinée aux seuls spécialistes ${ }^{3}$. Ainsi des saynètes comiques (Rochefort) ou des caricatures - le collectionneur-ours-en-cage vu par Champfleury: son habit est usé aux omoplates par le frottement de la chaise, «comme les aliénés bridés dans le corset de

2. «Une revendeuse» (publié dans L'Éclair, juin 1852) dans le recueil Une voiture de masques (1856).

3. Henri Rochefort, Les Petits Mystères de l'hôtel des ventes, [1862] (recueil d'articles du Charivari); Champfleury, L'Hôtel des commissaires-priseurs, 1867 (recueil d'articles de La Vie parisienne). 
force, qui, de même que les animaux des ménageries, se créent des récréations bizarres pour oublier leur liberté perdue»-, cohabitent avec un discours didactique, encore souvent parodique chez Champfleury («Dictionnaire à l'usage des connaisseurs qui ne s'y connaissent pas»), plus sérieux chez Rochefort, qui cite des articles de lois relatifs à la profession de commissaire-priseur, recommande nominativement quelquesuns d'entre eux, en indiquant leur notoriété, leur spécialité et leur tempérament.

En 1867, Champfleury prenait déjà ses distances, dans la préface du recueil, avec des textes publiés au début de la décennie et rendant compte d'un milieu découvert à la fin des années 1840. En cette même année 1867 paraît dans le Paris-Guide un article sur l'Hôtel Drouot de tonalité bien différente, signé de Philippe Burty, spécialiste des ventes d'art à la Gazette des Beaux-Arts: les salles de vente ne sont plus chez lui des tréteaux de foire. En outre, Champfleury évoquait un monde clos, une «franc-maçonnerie du bric-à-brac»; Burty souligne quant à lui la diversité des publics: «des ministres du dernier règne, des critiques d'art et de théâtre, des financiers billionnaires, des chanteurs célèbres en disponibilité, des dames du meilleur monde et de l'autre aussi, des marchands arrivés tout exprès de Londres, d'Amsterdam, de Vienne». L'hôtel des ventes est devenu, au même titre qu'une salle de spectacle lors d'une première représentation (la comparaison est de Burty), un lieu exemplaire de la capitale, qui offre un échantillon choisi de ses habitants. Dans le Paris-Guide, la seule variété excentrique de collectionneur qui demeure, c'est celle du bibliophile, croqué par Jules Janin dans le chapitre «Physiologie de Paris». Les autres ont l'honneur d'une mention nominative dans la première section, «La science L'art». L'inventaire des collections privées de la capitale fait suite aux descriptions de musées: elles ont désormais la même valeur d'utilité publique. Depuis les années 1840 , les collectionneurs ont donc acquis une indéniable légitimité, qui les place non plus en marge, mais à la tête de la société civile.

Pour finir avec le genre des chroniques de l'hôtel des ventes, on peut finalement considérer le cas de Paul Eudel, qui le reprend à son compte vers la fin du siècle (1882-1891 ${ }^{4}$. Il s'inscrit bien, par l'intermédiaire de son premier préfacier, dans la filiation de Champfleury et de Rochefort, qui s'inspiraient du pittoresque du lieu. Mais Eudel donne surtout des comptes rendus des ventes. Dans le troisième volume, il avoue d'ailleurs son vrai modèle, le Trésor de la curiosité de Charles Blanc, recueil érudit de catalogues de l'Ancien Régime, et renchérira en publiant en 1891 une Table des noms, transformant son ouvrage en guide d'achat et de vente. Quand il confie en 1885 la préface du quatrième volume à Champfleury, celui-ci ne peut que constater la distance parcourue: la collection, autrefois «jouissance intime de chat guettant une souris », est maintenant «une spécialité étudiée de près dans ses diverses ramifications», et le discours ironique et ludique est devenu un propos didactique pour «la foule des amateurs qui va s'augmentant d'année en année».

\section{L'excentrique: un rôle littéraire réduit après 1870}

Après 1870, la figure du collectionneur excentrique n'est plus tenable, parce qu'elle a perdu toute valeur référentielle: tout le monde (ou à peu près) collectionne, la collection, d'une déviance, est devenue une mode et une norme. L'ancien stéréotype

4. L'Hôtel Drouot en 1881, 1882; puis L'Hôtel Drouot et la curiosité en 1882, 1883 (un volume annuel jusqu'en 1889; index pour l'ensemble des volumes publiés sous le même titre en 1891). 
est alors, soit dénoncé dans des textes qui soulignent la nouvelle donne ${ }^{5}$, soit mis à distance, considéré comme un pur motif, et soumis à une élaboration littéraire au second degré.

L'exemple le plus simple en est donné par une comédie en un acte d'Ernest d'Hervilly (Le Bibelot, 1877), où l'intrigue amoureuse, respectée dans sa situation et son vocabulaire, est supplantée dans son objet par la quête du bibelot. Or le personnage du collectionneur est brossé à peu de frais par quelques expressions: "Voilà un terrible excentrique! C'est un monomane!» Ces termes apparaissent ainsi comme les traits définitoires du type, qui n'est plus qu'un rôle de théâtre, dépourvu même de ses caractéristiques physiques, sa laideur et son costume fatigué ( «Il n'a rien de hideux d'ailleurs [...] et puis, il est honorablement vêtu.»)

L'existence désormais purement fictive du personnage apparaît aussi dans une chronique de 1885 de Mirbeau. La légendaire obsession du collectionneur permet de le transformer en bandit féroce ou en amoureux transi: "les tapisseries lui ont des traîtrises de femme; il y a dans les dentelles des douleurs qui le font saigner; il languit pour un bout d'étoffe qui le fuit; il meurt pour un plat écorné qui ne veut pas de lui ». Le collectionneur réel en revanche (en la personne de Du Sommerard) est un personnage bien plus ordinaire et plus fade, une dupe fréquente (nombre des objets de Cluny sont des faux avérés, selon Mirbeau), et tout le contraire d'un héros de roman: en dénonçant ainsi la trivialité du réel, en abaissant aussi la figure de Du Sommerard, pourtant emblématique des premiers temps du collectionnisme, Mirbeau renvoie au mythe et à l'imaginaire littéraire la figure du collectionneur monomane ${ }^{6}$.

Rôle de théâtre, héros conventionnel, le collectionneur excentrique est réduit à la fonction de pure instance discursive dans deux textes écrits par Charles Cousin en 1878 (Voyage dans un grenier) et 1887 (Racontars illustrés d'un vieux collectionneur). L'auteur, bibliophile notoire, reprend la tradition du récit excentrique: le premier titre démarque Le Voyage autour de ma chambre de De Maistre, la structure dialoguée reproduit les rôles de Jacques le fataliste (le maître s'appelle ici le Tocqué) et dans le volume de 1887, l'Avis au lecteur précise le public visé: «confrères en toquade [...] curieux enragés, maniaques très précieux». Les chapitres sont autant de descriptions détaillées de pièces de collection, mais cette fois réelles, en la possession de l'auteur et reproduites par l'illustration. Le livre est en fait un catalogue d'œuvres d'art qui n'emprunte au genre fantaisiste que sa mise en forme, et le masque du collectionneur excentrique, qu'endosse ici l'auteur, est bien donné comme un héritage littéraire, qui concilie humour et érudition.

\section{Les nouveaux convertis}

Jusque dans les années 1860 , c'est le vrai collectionneur, le passionné qui est moqué ; à partir des années 1870 , ceux qui sont visés par l'ironie, la satire, voire le pamphlet, ce sont au contraire les faux collectionneurs, ceux dont la collection n'est plus que le moyen d'une ambition. La collection, critiquée comme pratique déviante,

5. Edmond Bonnaffé dans ses Causeries sur l'art et la curiosité (1878) dénonce comme périmée la physiologie d'Horace de Viel-Castel, qu'il cite sans nommer son auteur: «Ces excentriques dont on riait si bien, qu'un homme d'esprit appelait naguère des monomanes, martyrs de l'idée fixe, thésauriseurs féroces et sans pitié, dépeceurs de la France monumentale et artistique» sont maintenant considérés comme des sauveurs du patrimoine national.

6. Mirbeau, «Bibelots», Le Gaulois, 4 mars 1885. 
l'est désormais comme pratique déviée: puisque tout le monde collectionne, une discrimination s'impose, selon des critères qui portent d'abord sur les objets acquis (le vrai et le faux, l'ancien et le moderne, le rare et le commun, le beau et le laid), mais sanctionnent ensuite le choix du collectionneur, son goût, ses connaissances, qu'on rapporte finalement à ses origines et à ses motivations sociales ${ }^{7}$.

De sceau négatif univoque, la collection est devenue système de signes polysémiques invitant à un décodage sociologique avant la lettre, et le discours critique aura pour objet de révéler les différentes stratégies sociales dissimulées sous cette pratique généralisée. Certains y chercheront même l'identité esthétique et morale de l'époque tout entière. Pour Mirbeau en 1885, pour le dessinateur Albert Robida en 1888, la généralisation de la collection témoigne de l'incapacité du XIX ${ }^{e}$ siècle à s'inventer un style propre, ce qui n'est pas sans implications morales (c'est le règne du faux qui s'installe: «ce siècle est voué à la copie et à la reconstitution » ${ }^{8}$ ), ou politiques (une ville sans art et sans âme se profile à l'horizon: «Collectionnez [...] pour tâcher de ne pas voir les platitudes toutes neuves, les rangées de boîtes carrées qui constituent nos villes modernes $\left.[\ldots]{ }^{\prime}{ }^{9}\right)$. D' autres dénoncent le matérialisme qui a remplacé, avec le déclin des religions, une spiritualité défunte ${ }^{10}:$ avec l'accumulation de biens matériels (sinon les originaux hérités du passé, du moins leur moderne contrefaçon), c'est la société de consommation qui fait ses débuts en Europe, qui restaure, qui achète et qui entasse, avant d'apprendre à détruire et à jeter ${ }^{11}$.

Les textes qui se livrent à cette critique des faux collectionneurs après 1870 sont essentiellement des chroniques journalistiques ou des romans (réalistes ou psychologiques, pour reprendre les étiquettes de l'époque); plus ou moins virulents, les uns et les autres ont en tous les cas abandonné le discours parodique et ludique des physiologies, des contes, des récits excentriques. Ils entendent témoigner d'une réalité sociale, qu'ils dénoncent, au lieu de la percevoir dans un jeu de déformation et de grossissement. Chez les personnages croqués ou plus longuement campés, la collection n'est plus qu'une facette, une façade ou un moment. Elle importe moins que l'ambition qui la motive, et qu'il faut mettre au jour.

En 1840, aux côtés du «collectionneur pur-sang», le seul authentique, VielCastel mentionnait déjà deux autres espèces (de «faux» collectionneurs donc, chez lesquels la collection sert d'autres motivations): le «collectionneur brocanteur» et le «collectionneur par mode» ou «fashionable». Le premier, qui n'achète que pour revendre et réaliser un gain, disparaît assez vite des classifications : encore présent en 1861 dans l'Annuaire des amateurs et des artistes de Lacroix ${ }^{12}$, on ne le rencontre plus après 1870 , parce que la valeur symbolique de la collection, en termes de reconnaissance sociale, vaut plus désormais que sa valeur financière. Le «collectionneur

7. Les articles de Maupassant dans Le Gaulois et Gil Blas au début des années 1880 ont particulièrement recours à ces critères.

8. Mirbeau, «Bibelots», art. cité.

9. Albert Robida, Le XIX' siècle, 1888 («Les tribulations d'un homme de goût qui n'en avait pas »).

10. Voir le Préambule de Paul Ginisty, Le Dieu bibelot. Les collections originales, 1888.

11. Voir les excellentes analyses de Janell Watson, Literature and Material Culture from Balzac to Proust. The Collection and Consumption of Curiosities, Cambridge University Press, 1999.

12. «L'art et les artistes en $1860 »:$ :On ne rassemble plus une collection pour l'avoir, pour en jouir, pour s'en faire honneur, mais pour la vendre et pour gagner dessus.» 
par mode» à l'inverse se répand à partir de cette date. Au début des années 1860 , Lacroix ne le considère pas encore comme une figure caractéristique de l'époque, puisqu'il lui cherche des antécédents au siècle précédent: ce sont «les vaniteux que Watelet dit être des possesseurs de collections, qui s'en occupent vivement lorsqu'ils les font admirer, et les oublient lorsqu'ils sont seuls avec elles " ${ }^{13}$. Mais le type est déjà bien présent, comme l'atteste le personnage d'une comédie vaudeville tirée du livre de Rochefort sur l'hôtel Drouot ${ }^{14}$, Léopold Grandsécot, «jeune gandin»: «Moi, monsieur, j'ai horreur des curiosités», mais «quand on n'a pas pour 30000 francs de bibelots chez soi, les femmes vous méprisent; alors je suis en train de me monter une collection, pas pour moi, mais pour elles.»

Le personnage affiche ici sa véritable motivation (collectionner pour être à la mode et séduire) avec un cynisme si brutal qu'il en est comique. Les collectionneurs mis en scène après 1870 seront en général plus discrets, mais dénoncés par un même détachement à l'égard des objets assemblés, qui vaudront surtout pour leur conformité au goût ambiant. La marque de ces collectionneurs mondains sera donc paradoxalement le manque d'un goût propre. On la trouve chez Guy de Lissac, jeune élégant du «roman parisien» de Claretie, Monsieur le Ministre (1881). Son logement pourrait tout aussi bien être «l'appartement d'une femme à la mode» ou «la petite maison d'une jolie fille», il est plus l'œuvre de son tapissier que de son propriétaire. De la même façon, le collectionneur de la nouvelle génération mis en scène par Robida, par opposition au collectionneur de 1830, farouchement attaché à des objets dont la poussière dit l'ancienneté et l'immuabilité dans la demeure, constitue et revend successivement plusieurs collections, sans parvenir à s'attacher à aucune. Sa femme collectionne également, et la féminisation de la fonction, comme son déploiement sur l'échelle sociale (jusqu'aux prostituées), dit la vulgarisation de la pratique («Les actrices ont presque toutes la rage de bibeloter; tous les hôtels particuliers semblent des musées encombrés de saletés séculaires. " ${ }^{15}$ ) Sa spécificité s'efface, jusqu'à se confondre avec la décoration d'intérieur: les différentes étapes de la praxis de la collection ${ }^{16}$ (la quête, l'acquisition, la restauration, l'exhibition..., exemplairement illustrées dans les récits de collectionneurs traditionnels, comme Balzac ou Champfleury, et qui témoignaient de leur investissement affectif), disparaissent ici, déléguées à un professionnel (le tapissier). La collection devient un ensemble que l'on achète tout fait; ses composantes, comme ses propriétaires, semblent interchangeables ${ }^{17}$. Dans la chronique ou le roman, ces derniers fournissent des personnages sans beaucoup de relief.

Plus saillants seront ceux dont la collection doit manifester non le conformisme ou l'élégance «bon ton», mais une réussite sociale chèrement acquise. Les journalistes ou romanciers creusent alors volontiers l'écart qui existe entre leur milieu d'origine (la bourgeoisie d'affaires, l'industrie, la banque) et ses valeurs (l'utilité, le profit), d'une part, et leurs prétentions artistiques, d'autre part: leur rôle de collectionneur

13. Ibid., 1860, «Observations sur la tableaumanie».

14. Henri Rochefort et Albert Wolf, Les Mystères de l'hôtel des ventes, comédie vaudeville en trois actes, 1863.

15. Maupassant, «Bibelots», Le Gaulois, 22 mars 1883.

16. Dégagées par P.-M. de Biasi à propos du Cousin Pons («Système et déviances de la collection à l'époque romantique ", Romantisme, $\mathrm{n}^{\circ} 27,1980$ ).

17. Face à ce nivellement, l'originalité sera dans le choix d'objets nouveaux ou incongrus: jouets, pipes, timbres-poste, coquilles ( $P$. Eudel, Collections et collectionneurs, 1885), cannes, clefs et serrures ( $P$. Ginisty, Le Dieu bibelot. Les collections originales, ouvr. cité). 
leur sera d'autant plus cher, et ils le joueront avec d'autant plus d'éclat. La scène qu'esquisse Maupassant dans une de ses chroniques («Tout bourgeois ayant gagné dix mille francs de rentes dans l'industrie encombre sa salle à manger de ces affreuses assiettes normandes, peinturlurées ignoblement, qu'on vend maintenant au prix de la vaisselle plate, et il montre avec orgueil aux invités des vases ébréchés et ridicules achetés fort cher et valant, en vérité, fort peu.» ${ }^{18}$ ), - qu'avait avant lui racontée Champfleury avec les mésaventures de l'agent de change Bretoncel ${ }^{19}$, , se retrouve, sous une forme moins caricaturale et moins crue, dans les romans de Bourget ou d'Edith Wharton, et s'incarne par excellence dans le stéréotype du collectionneur américain, richissime et ignare, et soucieux surtout de perpétuer son nom ${ }^{20}$.

Autre exhibitionnisme bruyant, celui du petit bourgeois, du provincial, qui pose en spécialiste et se pique d'érudition. Ici, il ne s'agit même pas d'une ascension sociale à légitimer, mais, dans un milieu restreint et fermé, de se donner la respectabilité de préoccupations «scientifiques». C'est pourquoi les deux cloportes de Flaubert ne jouent pas tant au collectionneur qu'à l'archéologue. De même que pour leurs précédentes expériences, ils veulent donner un support livresque, une motivation scientifique à leur activité. Ils ne cherchent pas le beau, mais l'ancien, qui porte historiquement témoignage, et leur permette à leur tour, sinon d'écrire, du moins de dire l'histoire (c'est le sens de la visite qu'ils font de leur prétendu musée, où ils ont pour chaque pièce un commentaire) ${ }^{21}$. Cette orientation érudite de la collection et ses prétentions scientifiques seront également épinglées par Maupassant:

L'amateur d'antiquités aime tout: tout ce qui est vieux, tout ce qui est rare, tout ce qui est laid. Il s'extasie devant les ébauches informes des ouvriers primitifs, il pousse des cris en face des hideuses poteries de nos ancêtres naïfs; il sait, certes, il sait au juste à quelle époque fut fabriquée cette grossière statuette de faïence, et il en connaît le prix exact; et il la préfère à quelque ravissante ébauche en terre d'un artiste moderne. ${ }^{22}$

\section{Les spécialistes}

Face à la multitude des collectionneurs nouveaux venus dans le monde de l'art, se dressent les professionnels de la collection, historiens d'art ou chroniqueurs spécialisés, auteurs d'ouvrages didactiques, qui vont proposer des outils aux néophytes et manifester le renversement des valeurs associées à l'idée de collection.

Le discours érudit sur la collection existe depuis longtemps, centré plus volontiers sur les objets que sur les hommes, à l'inverse du discours ironique des physiologies. Néanmoins, par les aperçus historiques sur les amateurs du passé, par les préambules qui dessinent la physionomie d'un public restreint et choisi, une image du collection-

18. Maupassant, «Bibelots», art. cit.

19. «Les trouvailles de M. Bretoncel», conte repris dans L'Hôtel des commissaires-priseurs, ouvr. cité.

20. Bourget, «La Dame qui a perdu son peintre», 1910; Edith Wharton, Vieux New York, 1924 («L'aube mensongère (les années 1840)»).

21. Flaubert: Bouvard et Pécuchet (rédaction 1874-1881), chap. IV.

22. Maupassant, «Bibelots», art. cité. La qualité en moins, Bouvard et Pécuchet ressemblent aussi au collectionneur provincial dépeint par Bonnaffé (Physiologie du curieux, 1881): «Le provincial collectionne tout, les tableaux, les fibules mérovingiennes, les terres cuites, les livres, les silex et les morions, les monnaies, les assiettes et les parchemins. Son cabinet est un bazar: Gouthières coudoie Donatello, Chilpéric est voisin de Louis XIV, Robespierre de Périclès, Palissy donne la main aux antédiluviens. Il a un assortiment varié, comme certains marchands de petites villes qui tiennent la papeterie, les denrées coloniales, la mercerie et un peu de musique." 
neur modèle s'esquisse, bien distincte des portraits déjà caricaturaux de La Bruyère. Le Trésor de la curiosité de Charles Blanc (1857-1858) est précédé d'une substantielle préface du collectionneur Adolphe Thibaudeau, qui brosse le portrait du curieux de l'Ancien Régime, homme ouvert, nullement exclusif mais sensible au beau sous toutes ses formes, membre d'un «cercle d'esprits d'élite» à divers échelons de la société, unis dans la même entente du plaisir des yeux et de l'art de vivre. Savoir et plaisir: le collectionneur est à la fois un connaisseur et un dilettante.

C'est ce qui apparaît plus nettement encore dans les Causeries d'un curieux publiées entre 1862 et 1868 par Feuillet de Conches. Le titre d'abord est révélateur : il met l'accent sur l'aspect informel du propos et l'apparente à une conversation familière. La lettre au collectionneur Chaix d'Est-Ange qui sert de préface précise ce choix: souci de plaisir plus que d'érudition («[...] je n'ai pas écrit pour écrire et pour faire montre de savoir; j'ai écrit pour suivre mon goût et mon penchant.»); auteur et lecteurs appartiennent à un cercle restreint d'amis unis par les mêmes goûts :

[Mes autographes] ont été pour vous, pour un petit cercle d'amis, pour moi-même, une source toujours vive de récréation et d'instruction facile [...]. Tels jadis ces innocents badinages, mêlés de sage philosophie, que se permettaient, aux heures de repos et d'enjouement, Lélius et Scipion Émilien, après avoir dénoué leur ceinture.

Par cette allusion au De Amicitia de Cicéron, c'est l'otium antique qui sert de modèle à la collection et au discours sur la collection.

Ces valeurs seront remises en cause après 1870. Dans ses Causeries sur l'art et la curiosité (1878), Edmond Bonnaffé semble s'inscrire dans la continuité de Feuillet de Conches. Cependant il récuse d'entrée de jeu les motivations égoïstes ou élitistes du discours érudit: «Mais ces précieuses reliques une fois recueillies, on n'entendait pas les réserver pour le régal exclusif de quelques délicats; il fallait en tirer un enseignement, l'éducation du public était encore à faire.» La collection n'est plus simplement un goût, elle est devenue une mission; en amont, «pour sauver, en dépit de certaines gens, les épaves de l'ancien temps »; en aval, pour instruire et éveiller à l'art toutes les couches sociales. Le collectionneur ne saurait avoir l'insouciance du patricien antique, il a désormais un devoir, qui l'apparente à l'apôtre ou au chien de terreneuve!

Même critique, plus acerbe peut-être, dans la présentation que donne Ernest Bosc de son Dictionnaire de l'art, de la curiosité et du bibelot (1883), ouvrage de vulgarisation, destiné non plus à une élite déjà informée mais aux nouveaux collectionneurs, et qui lui semble combler une lacune: il existe certes des livres sur la curiosité, mais ils sont assez pointus,

ils n'embrassent pas d'une manière générale, d'une manière assez encyclopédique, le vaste domaine de la curiosité. C'est qu'en effet, c'était un bien gros travail. [...] Or le curieux est nécessairement un homme riche et de loisir, c'est-à-dire un gentleman qui aime beaucoup à voir, à flâner, à toucher, à palper, à voyager sans souci d'aucune sorte ; la causerie entre amis, entre confrères, voilà son fait; $[\ldots]$ le travail chez le curieux... c'est là son moindre défaut.

On touche du doigt une modification radicale, où le travail devient une valeur qui supplante la valeur antique, humaniste puis aristocratique, de l'otium.

Le propos sur la collection tend ainsi à devenir nettement didactique: les guides, manuels et dictionnaires se multiplient: le discours érudit sur les objets, leurs caracté- 
ristiques techniques ou leur histoire, se double d'un discours second sur la praxis de la collection: qui est collectionneur? (Ris-Paquot commence à publier son Répertoire général des collectionneurs de la France et de l'étranger en 1882); comment faut-il collectionner, acheter, restaurer, exposer? (Le Livre des collectionneurs d'A. MazeSencier (1885) donne des conseils par types d'objets: la dinanderie, l'horlogerie, la céramique...) La forme la plus naïve et la plus explicite du genre apparaît dans un article de la dernière livraison du Japon artistique (mars 1891) sur «L'art de collectionner», où l'auteur, Marcus B. Huish, prodigue les recommandations les plus prosaïques (tenir compte de ses revenus, de l'espace disponible dans son habitation, de la présence éventuelle d'enfants), pour déterminer a priori le type d'objets à rassembler. La pratique de la collection ainsi objectivée, par un ensemble de règles préétablies, n'est plus la passion qui enflammait l'excentrique. Le collectionneur, bourgeois prudent, réserve à la collection une place circonscrite dans sa demeure; il suit pour cela les conseils des meilleurs professeurs; il se donnera ainsi l'illusion de contribuer à la formation ou à la préservation du patrimoine artistique du pays, et pourra afficher fièrement cette prétention, en ouvrant son petit musée aux amis, en prêtant aux expositions ${ }^{23}$.

\section{L'aristocrate collectionneur}

À la fin du siècle apparaît dans les romans une figure qui récuse à la fois le banal conformisme des collectionneurs néophytes, et les prétentions dogmatiques, la visée utilitariste (éducation des goûts du peuple, formation des artistes, inspiration des industries d'art), des spécialistes de la collection: c'est celle de l'aristocrate collectionneur, qui affirme sa supériorité en vertu d'une pratique plus ancienne et partant plus authentique, fondée sur d'autres valeurs que le savoir ou la mode. La collection est chez lui un héritage et une tradition, elle partage son cadre de vie, elle est chargée d'une histoire familiale. On est loin de l'engouement récent de l'homme d'affaires, ou du savoir livresque du spécialiste.

C'est surtout ce dernier que l'aristocrate conteste, le premier étant d'emblée hors jeu. Dans la nouvelle de Bourget «La dame qui a perdu son peintre» (1910), les trois types apparaissent, confrontés à un problème d'attribution: le grand couturier soucieux d'une plus grande respectabilité, l'expert, disciple de Morelli, qui l'accompagne en Italie pour l'aider dans le choix de ses primitifs, et le noble italien, entouré de «trésors amassés dans sa maison par plusieurs générations de riches patriciens »; or le grand bourgeois se révèle plus sensible à l'art que son conseiller, enfermé dans ses enquêtes érudites ( «des semaines de fouilles dans les paperasses, des établissements de dossiers, des expertises d'écriture lettre par lettre, point par point, d'infinies comparaisons»). Mais c'est finalement le sens inné du beau chez le noble qui triomphe des laborieuses conclusions de l'expert, lequel persiste grotesquement dans son erreur.

Les valeurs aristocratiques de la collection, défendues par Bourget, sont néanmoins menacées. Le personnage qui les incarne appartient à une race en voie de disparition, sa fin prochaine annonce aussi celle de tout un monde. Le marquis de

23 Le collectionneur devient un homme public, alors que l'excentrique de 1840 se renfermait dans sa demeure. La Physiologie du curieux de Bonnaffé souligne bien cet aspect nouveau, en mettant notamment en scène les différents types du prêteur, du propriétaire exposant, du visiteur. La rédaction d'un catalogue, l'aménagement de la maison en musée deviennent des étapes codées de la pratique: voir Robida, art. cité, et Eudel, Collections et collectionneurs (1885), «Aimé Desmottes». 
Claviers-Grandchamp dans L'Émigré (1907) découvre finalement qu'il est sans héritier, que son fils est l'enfant naturel d'un grand bourgeois. Dans «L'adoration des mages» (1897), le personnage du collectionneur, un noble en révolte contre le monde moderne, était déjà un vieillard solitaire. Dans «Monique» (1901), où il n'y a pas à proprement parler de collectionneur, mais un vieil artisan d'art, restaurateur de meubles anciens, et dépositaire des valeurs traditionnelles du rapport à l'art, voit aussi partir celui qu'il désirait pour successeur. Dans le roman d'Henry James, Les Dépouilles de Poynton (1897), la collectionneuse n'est pas une aristocrate, mais sa collection est présentée comme l'œuvre de toute une vie: or elle perd également son fils, dont s'empare une jeune arriviste, absolument fermée à l'art, et dans un incendie accidentel, sa demeure s'envole en fumée.

À la fin du $\mathrm{XIX}^{\mathrm{e}}$ siècle et au début du $\mathrm{XX}^{\mathrm{e}}$, on observe donc un retour aux valeurs aristocratiques de l'art et de l'otium dans la pratique de la collection, incarné cette fois par un type romanesque bien marqué, celui de l'aristocrate collectionneur, qui n'est plus tout à fait le curieux de l'Ancien Régime, plus sensible à la menace qui pèse sur sa race et sur ses possessions. Face à lui se dressent les nouveaux collectionneurs, spécialistes, experts et professeurs de la Troisième République, ou leurs élèves, venus à l'art sur le tard, au terme d'une réussite économique et sociale. De sorte que deux types de discours «sérieux» et partisans s'affrontent désormais sur les ruines du discours ironique et parodique: l'un, républicain, souligne la valeur scientifique et pédagogique de la collection, l'autre, aristocratique, privilégie sa valeur artistique et sa fonction strictement privée. Ces deux options semblent s'incarner en deux types de lieu, l'habitation et le musée. Le musée, institution du XIX ${ }^{e}$ siècle, lieu de sauvegarde et école de l'art pour les uns, froide nécropole par les autres; la maison du collectionneur, ensemble plus complexe que le cabinet particulier qui concentrait jadis les pièces de collection, lesquelles ont désormais envahi tout l'espace privé. Resterait à examiner plus précisément les discours sur ces nouveaux espaces, qui occupent à la fin du siècle le devant de la scène romanesque, y déploient leurs architectures et leurs vitrines, très loin des arrière-plans poussiéreux qui servaient de décors aux vieux excentriques. Dans les romans de la fin du siècle, la collection est devenue, aux côtés du collectionneur, un actant essentiel, dont le devenir fait l'enjeu de l'intrigue ${ }^{24}$.

(Université de Paris III)

24 Voir notamment E. de Goncourt, La Maison d'un artiste (1881), J.-H. Rosny, Le Testament volé (1905), Bourget «La seconde mort de Broggi-Mezzastris» (1904) et L'Émigré (1907), et sur le musée nécropole, les travaux de J.-P. Guillerm, notamment son article «Matières et musiques» dans Des mots et des couleurs, textes réunis par Ph. Bonnefis et P. Reboul, Université de Lille III, 1979. 